\title{
Onward from solar convection to dynamos in cores of massive stars
}

\author{
Juri Toomre \\ JILA \& Dept of Astrophysical and Planetary Sciences, University of Colorado, Boulder, CO \\ 80309-0440, USA \\ email: jtoomre@jila.colorado.edu
}

\begin{abstract}
We reflect upon a few of the research challenges in stellar convection and dynamo theory that are likely to be addressed in the next five or so years. These deal firstly with the Sun and continuing study of the two boundary layers at the top and bottom of its convection zone, namely the tachocline and the near-surface shear layer, both of which are likely to have significant roles in how the solar dynamo may be operating. Another direction concerns studying core convection and dynamo action within the central regions of more massive $\mathrm{A}, \mathrm{B}$ and $\mathrm{O}-$ type stars, for the magnetism may have a key role in controlling the winds from these stars, thus influencing their ultimate fate. Such studies of the interior dynamics of massive stars are becoming tractable with recent advances in codes and supercomputers, and should also be pursued with some vigor.
\end{abstract}

Keywords. Sun: tachocline, Sun: near-surface shear layer, Sun: dynamo action, stars: massive star convection

\section{Coupling of stellar convection, rotation, magnetism and winds}

The last decade has seen a major resurgence of interest in many university departments concerning the structure and evolution of a broad range of stars. Contributing to this are the abundant successes in detecting extra-solar planets, and thus the need to understand in some detail the dynamical properties of the central stars in such systems as one ponders the possibilities of life elsewhere. Similarly, significant observational advances in spectropolarimetry and in Doppler imaging are permitting deductions about differential rotation and magnetic structures at the surfaces of a variety of stars, thus calling out for detailed theoretical interpretations and explanations. Further, asteroseismology is showing its ability to infer interior properties of stars, and when carried out on very large samples as permitted with Kepler observations, is raising many fascinating puzzles that require major new theoretical efforts to analyze the evolving interior dynamics of stars. Central to all of these topics is the need to understand the coupling of convection, rotation, shear and magnetism in stellar settings, which raises formidable theoretical challenges since the flows and structures are highly turbulent and involve a vast range of physical scales.

Convection plays many roles in the lives of stars. Moderate and low mass stars on the main sequence (when they burn hydrogen in their cores) possess convective envelopes and radiative interiors, whereas more massive stars have convective cores and radiative envelopes. Such turbulent convection in the outer envelopes of the less massive F, G, K and $\mathrm{M}$ stars, when influenced by the rotation of the star, can yield magnetic dynamo action that is likely responsible for much of the magnetic activity that is observed in their atmospheres. The effects of dynamo action in the cores of more massive $\mathrm{O}, \mathrm{B}$ and A stars is less certain, for their extensive radiative envelopes may be effective in hiding contemporary magnetic field building as it proceeds. Thus the magnetic spots that are 
observed to rotate into view on some of these stars may well be mainly a signature of surviving primordial fields. However, if sufficiently strong and thus buoyant magnetic structures can be built by core dynamos, then such fields have the possibility of rising to the surface to be detected. Whether the contemporary core fields get out or not, they are likely to have an important role in the final stages of stellar evolution. Late in their lives as the massive stars expel their envelopes to expose their very hot cores and thus leave behind white dwarfs, or even after supernova explosions that leave behind neutron stars, that dynamo action may help to explain or contribute to the very strong magnetic fields that are observed in the remnants.

As another issue, the presence of strong magnetic fields close to the surface of all stars may have pivotal effects upon the strength and character of stellar winds that both carry away mass and angular momentum as the stars evolve. This may well influence whether some of the massive stars have lost enough mass to end their lives relatively quietly as white dwarfs, or whether they have a more fiery end as a core collapse supernova that leaves behind a neutron star or even a black hole. The moral from all this is that stellar convection, rotation, magnetism and winds are all closely linked in the life stories of most stars. The past decade or two has seen substantial progress in beginning to deal with these interlinked and highly nonlinear dynamical processes, as this conference is amply revealing. These decades have also provided splendid high-resolution imagery from Hubble Space Telescope that emphasize the richness of structure in the winds that must be attributable to both rotation and magnetism. It may be appropriate to reflect upon a few of these developments that should be pursued with vigor in the near future.

\section{Ascending role of computational astrophysics}

Computational astrophysics has become the third arm of research that complements both basic theory and observations, attaining this position through the ability to conduct ever more realistic 3-D simulations due to rapid advances in both supercomputers and in codes and algorithms that can exploit such machinery. These advances have enabled the simulations of convection and dynamo action in the outer envelope of the Sun and in their more rapidly rotating younger relatives, as discussed in graphic detail in our meeting. These are having a pivotal role in coming to understand the elements involved in achieving the differential rotation of such stars and of the vigorous magnetic dynamo that can result, even yielding magnetic fields that can take the form of striking wreaths that persist or ones that reverse their sense cyclically or episodically. The challenge here is to get the modeling of global-scale convection within spherical shells, such as carried out with the anelastic spherical harmonic (ASH) code, to regimes with ever smaller diffusivities and thus more turbulent conditions. Further, one needs more realistic treatments of the two boundary layers that are present in the Sun, namely the tachocline of rotational shear as revealed by helioseismology at the base of the convection zone and the near-surface shear layer just below the surface.

\section{Solar tachocline}

Dealing with the tachocline requires resolving penetrative convection entering a region of very stable stratification in which the downward-directed plumes can excite a rich medley of gravity waves, with all of this somehow establishing the pronounced rotational shear that is observed. Keeping that shear from gradually spreading into the deeper radiative interior that is deduced to be in solid-body rotation may well require magnetic fields, possibly involving very modest fields of primordial origin that survive in the deep 
interior, along with a magnetic boundary layer within the tachocline itself to isolate the deep from the fields and shear built within the convection zone itself. Amidst all such needs to keep that boundary layer seemingly narrow, this tachocline is likely to be the key factor in the reversing cycles of erupting magnetism observed as sunspots. How the tachocline is able to order and shear toroidal magnetic fields into structures that become buoyantly unstable in a self-consistent manner is the dominant challenge to dynamo theory. This has a reasonable hope of being sorted out through global 3-D simulations in roughly the next five years or so. It requires focused attention to attain realistic solar stratifications at high spatial resolution and much lower diffusivities in order to get into regimes that have a likelihood of building sufficiently strong and ordered toroidal fields. A recent new ingredient is that the lower reaches of the convection zone may be able generate leaky wreaths of fairly strong toroidal fields that already have a preferred different sense in the two hemispheres, as we have seen for younger Suns rotating more rapidly (Brown et al. 2010, 2011). This behavior may well carry over to the Sun itself as diffusive processes are lessened, with downward magnetic pumping serving to bring fields into the tachocline region to be further amplified and the more random elements gradually eliminated (Browning et al. 2006). It will be fascinating to discover just what sets the roughly 11-year periods for reversals, whether it be the meridional circulation times favored by flux-transport and some mean-field models, or possibly intrinsic competing generation processes in the wreaths or in the tachocline itself. We are beginning to see 3-D global solar dynamo simulations that are now possessing cycles (Ghizaru et al. 2010; Miesch et al. 2011), and these serve to encourage much more work to understand their sensitivities.

\section{Solar near-surface shear layer}

Seeking to understand the origin and role of the Sun's near-surface shear layer raises challenges that are quite different from that of the boundary layer at the base of the convection zone. Here we have intense competition and likely collisions between the fast small scales of descending granulation and supergranulation, driven by rapid cooling at the solar surface, with the ascending and sweeping large scales of giant cells of convection. Those giant cells sense rotation strongly and are key to achieving the differential rotation seen in the bulk of the convection zone (Miesch et al. 2008). Yet such current 3-D global simulations of solar convection with ASH do not take into account the descending plume patterns from the surface, nor do the localized-domain surface convection simulations (Stein et al. 2009) account for ascending or shearing motions from the deep. Thus neither achieves a near-surface shear layer, though helioseismology reveals its rotational signature in roughly the outer $5 \%$ in radius quite clearly. The forbidding barrier to either local or global models is the vast range of spatial and time scales that are inherent in solar convection in its upper reaches. This may be overcome by turning to intermediate domain simulations in depth that focus on spherical shell segments, being informed from above by the statistics of the descending plume networks of supergranulation, and from below by the ascending and shearing flows of giant cells. The required simulation tools are now becoming available, as with the compressible spherical segment (CSS) code (Augustson et al. 2011b), and hold out the promise that properties of the near-surface shear layer and its multi-scale flow interactions may soon be capable of starting to be resolved. Such an approach will also be essential to devise new upper boundary conditions for the global simulations, as with ASH, that capture the more gradual flow decelerations and deflections that are likely to result as the rising giant cells meet the intense small scales from above. The inclusion of near-surface dynamics may have subtle influences on 

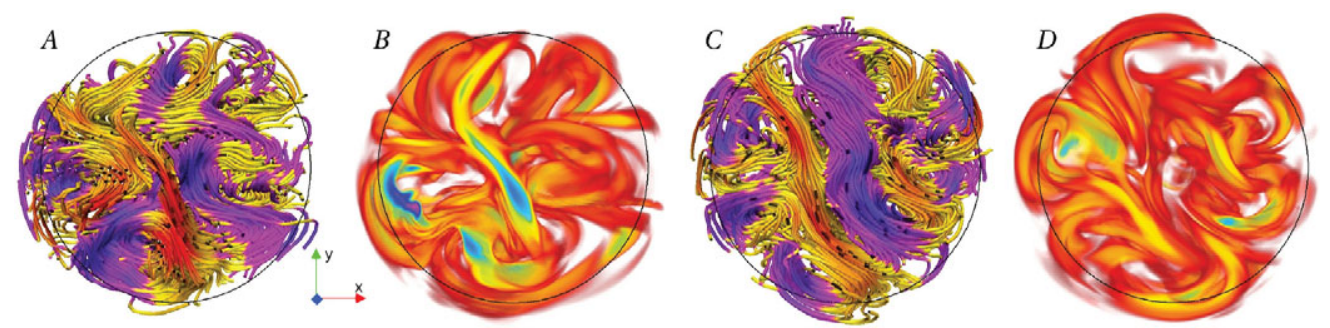

Figure 1. Evolving flow streamlines $(A, C)$ and magnetic energy density $(B, D)$ in the super equipartition A-type star convective core dynamo. Shown is the equatorial plane in two time instants separated by 50 days. Violet tones denote positive motions in the $y$-direction, and yellow tones negative motions. Regions of strong magnetic energy are shown in yellow/green tones. Large core-crossing flows carry broad swaths of magnetic field with them.

the character of differential rotation and meridional circulations achieved in the global modeling, and thus some account of the upper boundary layer must be pursued. Further, it is the near-surface shear layer that can now be probed in uninterrupted detail to study subsurface flows and their interaction with magnetism using local-domain helioseismology, as now feasible using Doppler and magnetic imaging data from the Solar Dynamics Observatory and its HMI and AIA instruments.

\section{Marching toward convection and dynamos in massive stars}

Stars more massive than about 1.5 solar masses (the A, B and O-type stars) possess radiative envelopes surrounding a convective core. Long suspected of harboring core dynamos, a subset of these massive stars also exhibit striking surface magnetism. These peculiar A- and B-type stars have strong patches of surface magnetism thought to arise where large-scale subsurface magnetic fields extend through the stellar surface. Such subsurface fields likely evolved from magnetic field initially threading the collapsing molecular cloud that formed the star. Our initial investigations into the nature of core dynamos of these massive stars focused on A-type stars lacking such an external field. In its absence, these core dynamos can generate magnetic fields with energies in near equipartition with the convective motions (Brun, Browning \& Toomre 2005). Recent work with ASH began to examine the coupling of such a convective core dynamo to a primordial magnetic field threading the radiative envelope. Dynamo action achieved in the presence of such a field is much stronger than that found in its absence, yielding magnetic energies roughly ten-fold that with no primordial field (Featherstone et al. 2009). This is a remarkable finding, for here one has achieved a super-equipartition dynamo state that was not thought to be realizable, and may well be characteristic of many core dynamos. The super-equipartition dynamo is characterized by the presence of four to six meandering convective rolls whose motions frequently cross the rotation axis, linking distant regions of the core as they do so (Figure 1). The effects of stellar rotation, here at $4 \Omega_{\odot}$, are a crucial element in building these structures. Strong axial circulations in the cores of these rolls lead to plunging events that couple the northern and southern hemispheres. These motions serve to advect magnetic field from regions of strong shearing and generation, mixing it throughout the bulk of the core. This work suggests that new initiatives should be undertaken in the next few years to explore the limiting strength of such toroidal fields, here already at about $500 \mathrm{kG}$, as less diffusive approaches are employed in codes such as ASH. Even stronger fields may become magnetically buoyant and have the 

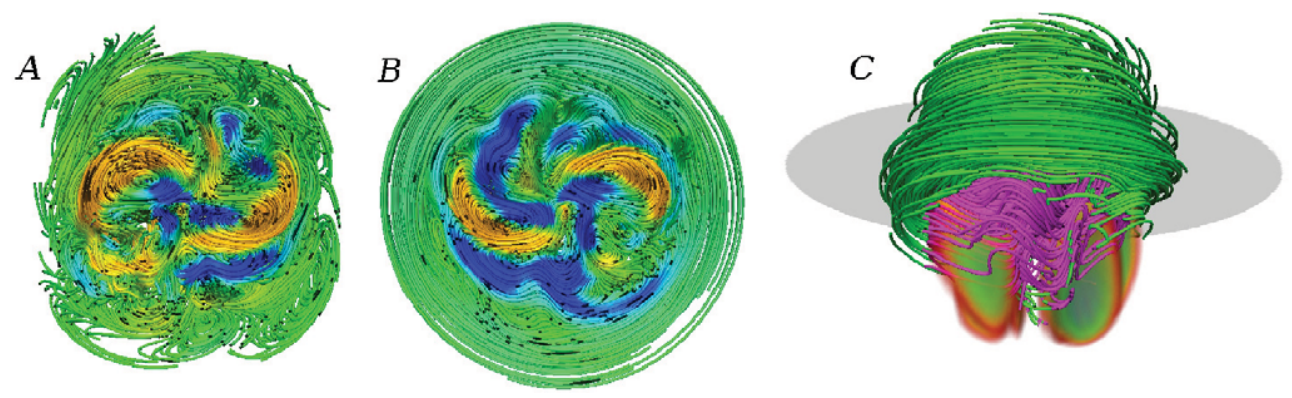

Figure 2. Convection and dynamo action in the core of a B star of mass $10 \mathrm{M}_{\odot}$ rotating at $8 \Omega \odot$. Shown in $(A)$ are a snapshot of velocity streamlines within the core, cut along the equator viewing the southern hemisphere. Rapid flows in yellow tones cross the core, and several columnar flows are evident. In contrast, $(B)$ shows the same of the northern hemisphere, which exhibits strong differential rotation. In $(C)$ are magnetic field streamlines in green and pink (for polarity) in perspective side view (equator shaded), emphasizing the dominance of magnetism in the northern hemisphere in this solution.

potential to erupt from the convective core toward the surface, thus providing a possible linkage between contemporary dynamo action in the deep with fields seen at the surface.

While $\mathrm{O}$ and B-type stars make up only a small fraction of the total stars in a typical galaxy, they are very luminous and have short lives. Indeed, a 10 solar mass B star has a luminosity about $7200 \mathrm{~L}_{\odot}$, and lives on the main sequence (fusion burning hydrogen in its core) for only about 15 million years (compared to 10 billion for the Sun). Many O and B stars exhibit strong stellar winds, and many end up in supernova explosions, enriching the interstellar medium with heavy elements. They thus influence star formation and galactic structure throughout their brief lives, thereby having critical roles in many of the processes that sustain and evolve their host galaxies. Understanding how the convective dynamics occurring within the cores of massive stars alters their lifetimes is of interest to many astrophysical communities. Since the exact conditions necessary for self-sustained magnetism in $\mathrm{O}$ and $\mathrm{B}$ stars is not known, we need detailed study of the coupling of the radiative zone and the convective core in $\mathrm{O}$ and $\mathrm{B}$ stars. If the core dynamos are able to get magnetic fields into the atmospheres of these stars, again by field possibly erupting from the core, then their winds may be influenced by the presence of such magnetic fields, both in terms of mass loss and stellar spindown. As our preliminary simulations of B star core convection are revealing (Augustson et al. 2011a), sustained dynamo action leading to strong magnetic fields within the core are indeed realized (Figure 2), but for the case shown here there are pronounced asymmetries in the field strengths achieved in the northern and southern hemispheres. Other rotation rates lead to more symmetric states. These studies are showing that its is now feasible to begin exploring the interior dynamics of massive stars in some detail through 3-D simulations. This subject is ripe for investigation given the codes and computers that are becoming available, but above all motivated by the realization that the massive stars are the dominant recyclers in any galaxy. The near future for such research looks most promising, as one has seen with both A and B stars, and thus the study of massive star dynamics may become one of the areas of vigorous study.

\section{Positive optimism that may be deserved}

All the work implicitly discussed briefly here has been carried out as joyful collaborations with many friends dealing with helioseismology and with solar and stellar convection 
and dynamo theory, a good number of whom are at this meeting. I thank them for their enthusiastic participation and wisdom in such work, and hope that the research investments made so far will continue to bear splendid fruit as the topic of astrophysical fluid dynamics (AFD) continues to mature and expand into areas that seemed overly challenging not so long ago. Coming to really understand the operation of the solar global dynamo in a reasonably self-consistent manner is getting tantalizing closer to hand, and we hope that this does not prove to be a shimmering mirage, given the surprises that highly turbulent systems are able to provide. Yet the Sun seems to know how to operate its magnetic cycles in a largely regular fashion, assuming that we can overlook the occasional hiccups that may be extended minima. It is but for us to try to sort out the elements involved in such dynamo action, with the ever enhanced computational resources providing us with experimental tools that may be up to the task. The presence of two boundary layers in the solar convection zone certainly warns us of subtle effects that need to be considered with some care, but the next five years holds out good promise that we may achieve substantial steps forward. The suggested investments to study interior dynamics within the more massive stars on the main sequence are in earlier stages, yet they benefit greatly from the tools and experiences that have come about from trying to understand the functioning of our nearest modest star. The $\mathrm{O}$ and $\mathrm{B}$ stars with their great luminosities and short lives, and often with strong winds, are now becoming quite tractable to detailed simulation. This may well become one of the renaissance research areas in AFD, complementing in many ways the intense efforts to understand how these stars late in their lives may come to explosive conclusions, but not always. The core convection and its dynamos may well provide keys to the masses that survive late in such stellar lives.

This research was supported recently by NASA Heliophysics Theory grant NNX08AI57G and by NSF Teragrid supercomputing resource grant TG-MCA93S005 and NASA project GID 26133.

\section{References}

Augustson, K. C., Brun, A. S., \& Toomre, J. 2011a, these proceedings

Augustson, K., Rast, M., Trampedach, R., \& Toomre, J. 2011b, J. Phys. Conf. Ser. 271, 012070

Brown, B. P., Browning, M. K., Brun, A. S., Miesch, M. S., \& Toomre, J. 2010, ApJ, 711, 424

Brown, B. P., Miesch, M. S., Browning, M. K., Brun, A. S., \& Toomre, J. 2011, ApJ, 731, 69

Browning, M. K., Miesch, M. S., Brun, A. S., \& Toomre, J. 2006, ApJ, 648, L157

Brun, A. S., Browning, M. K., \& Toomre, J. 2005, ApJ, 629, 461

Featherstone, N. A., Browning, M. K., Brun, A. S., \& Toomre, J. 2009, ApJ, 705, 1000

Ghizaru, M., Charbonneau, P., \& Smolarkiewicz, P. K. 2010, ApJ, 715, L133

Miesch, M. S., Brown, B. P., Browning, M. K., Brun, A. S., \& Toomre, J. 2011, these proceedings

Miesch, M. S., Brun, A. S., DeRosa, M. I., \& Toomre, J. 2008, ApJ, 673, 557

Stein, R. F., Georgobiani, D., Schafenberger, W., Nordlund, A. A., \& Benson, D. 2009, AIP Conf. Ser. 1094, 764 